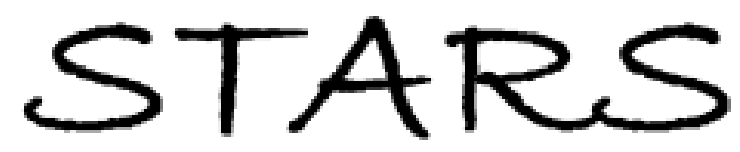

University of Central Florida

STARS

$1-1-2012$

\title{
Optical limiting and spectral stabilization in segmented photonic lattices
}

\author{
Matthias Heinrich \\ University of Central Florida \\ Falk Eilenberger \\ Robert Keil \\ Felix Dreisow \\ Eric Suran
}

See next page for additional authors

Find similar works at: https://stars.library.ucf.edu/facultybib2010

University of Central Florida Libraries http://library.ucf.edu

This Article is brought to you for free and open access by the Faculty Bibliography at STARS. It has been accepted for inclusion in Faculty Bibliography 2010 s by an authorized administrator of STARS. For more information, please contactSTARS@ucf.edu.

\section{Recommended Citation}

Heinrich, Matthias; Eilenberger, Falk; Keil, Robert; Dreisow, Felix; Suran, Eric; Louradour, Frédéric; Tünnermann, Andreas; Pertsch, Thomas; Nolte, Stefan; and Szameit, Alexander, "Optical limiting and spectral stabilization in segmented photonic lattices" (2012). Faculty Bibliography 2010s. 2735. https://stars.library.ucf.edu/facultybib2010/2735

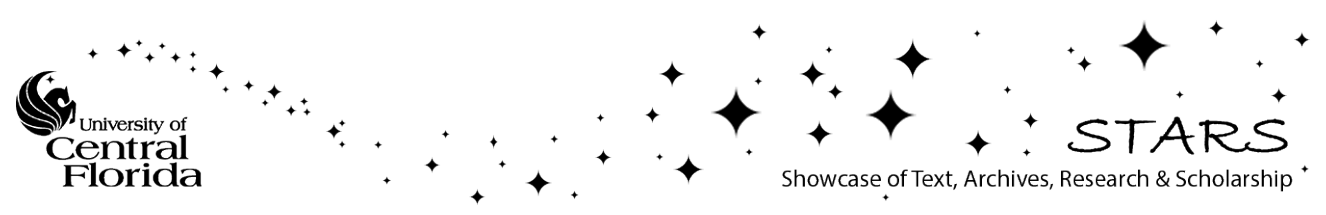




\section{Authors}

Matthias Heinrich, Falk Eilenberger, Robert Keil, Felix Dreisow, Eric Suran, Frédéric Louradour, Andreas Tünnermann, Thomas Pertsch, Stefan Nolte, and Alexander Szameit 


\title{
Optical limiting and spectral stabilization in segmented photonic lattices
}

\author{
Matthias Heinrich, ${ }^{1,2,}{ }^{*}$ Falk Eilenberger, ${ }^{2}$ Robert Keil, ${ }^{2}$ Felix Dreisow, ${ }^{2}$ \\ Eric Suran, ${ }^{3}$ Frédéric Louradour, ${ }^{3}$ Andreas Tünnermann, ${ }^{2}$ Thomas \\ Pertsch, ${ }^{2}$ Stefan Nolte, ${ }^{2}$ and Alexander Szameit ${ }^{2}$ \\ ${ }^{1}$ CREOL, The College of Optics $\mathcal{E}$ Photonics, University of Central Florida, Orlando, Florida \\ 32816, USA \\ ${ }^{2}$ Institute of Applied Physics, Abbe Center of Photonics, Friedrich-Schiller-Universität Jena, \\ Max-Wien-Platz 1, D-07743 Jena, Germany \\ ${ }^{3}$ XLIM Institut de Recherche, CNRS-Université de Limoges, Faculté des Sciences, 123 av. A. \\ Thomas, F-87060 Limoges, France \\ *matthias.heinrich@ucf.edu
}

\begin{abstract}
We propose photonic lattices with segmentation-based linear self imaging as integrated optical limiters. Starting from unity transmission in the linear regime, nonlinear delocalization leads to a continuous decrease of the overall transmission for increasing input powers. The diffractive propagation between input and output port offers the additional benefit of substantially decreased nonlinear spectral distortions. The functionality is demonstrated experimentally in a waveguide lattice realized in bulk fused silica with the femtosecond laser writing technique.
\end{abstract}

(C) 2012 Optical Society of America

OCIS codes: (190.4360) Nonlinear optics, devices; (190.6135) Spatial solitons; (190.5530)

Pulse propagation and temporal solitons.

\section{References and links}

1. B. J. Eggleton, B. Luther-Davies, and K. Richardson, "Chalcogenide photonics," Nature Photon. 5, 141-148 (2011).

2. T. Vo, J. Schröder, B. Corcoran, J. V. Erps, S. J. Madden, D.-Y. Choi, D. A. P. Bulla, B. Luther-Davies, M. D. Pelusi, and B. J. Eggleton, "Photonic-chip-based ultrafast waveform analysis and optical performance monitoring," IEEE J. Sel. Top. Quantum Electron. 18, 834-846 (2012).

3. H. Ji, M. Galili, H. Hu, M. Pu, L. K. Oxenløwe, K. Yvind, J. M. Hvam, and P. Jeppesen, " 1.28 -tb/s demultiplexing of an otdm dpsk data signal using a silicon waveguide," IEEE Photon. Technol. Lett. 22, 1762-1764 (2010).

4. L. W. Tutt and T. F. Boggess, "A review of optical limiting mechanisms and devices using organics, fullerenes, semiconductors and other materials," Prog. Quantum Electron. 17, 299 (1993).

5. J. W. Perry, K. Mansour, I.-Y. S. Lee, X.-L. Wu, P. V. Bedworth, C.-T. Chen, D. Ng, S. R. Marder, P. Miles, T. Wada, M. Tian, and H. Sasabe, "Organic optical limiter with a strong nonlinear absorptive response," Science 273, 1533-1536 (1996)

6. D. E. Spence, P. N. Kean, and W. Sibbett, "60-fsec pulse generation from a self-mode-locked ti:sapphire laser," Opt. Lett. 16, 42-44 (1991).

7. R. C. C. Leite, S. P. S. Porto, and T. C. Damen, "The thermal lens effect as a powerlimiting device," Appl. Phys. Lett. 10, 100-101 (1967).

8. A. Szameit and S. Nolte, "Discrete optics in femtosecond laser-written photonic structures," J. Phys. B 43, 163001 (2010).

9. F. Lederer, G. Stegeman, D. Christodoulides, G. Assanto, M. Segev, and Y. Silberberg, "Discrete solitons in optics," Phys. Rep. 463, 1-126 (2008).

10. Y. V. Kartashov, V. A. Vysloukh, and L. Torner, "Soliton Shape and Mobility Control in Optical Lattices," Prog. Opt. 52, 63-148 (2009).

\#174173 - \$15.00 USD Received 10 Aug 2012; revised 11 Sep 2012; accepted 12 Sep 2012; published 19 Nov 2012

(C) 2012 OSA

19 November 2012 / Vol. 20, No. 24 / OPTICS EXPRESS 27299 
11. D. Christodoulides and R. Joseph, "Discrete self-focusing in nonlinear arrays of coupled waveguides," Opt. Lett. 13, 794-796 (1988).

12. Y. Kivshar, "Self-localization in arrays of defocusing waveguides," Opt. Lett. 18, 1147-1149 (1993).

13. D. Christodoulides and E. Eugenieva, "Blocking and routing discrete solitons in two-dimensional networks of nonlinear waveguide arrays,” Phys. Rev. Lett. 87, 233901 (2001).

14. R. Keil, M. Heinrich, F. Dreisow, T. Pertsch, A. Tünnermann, S. Nolte, D. N. Christodoulides, and A. Szameit, "All-optical routing and switching for three-dimensional photonic circuitry," Sci. Rep. 1, 94 (2011).

15. J. Yang, P. Zhang, M. Yoshihara, Y. Hu, and Z. Chen, "Image transmission using stable solitons of arbitrary shapes in photonic lattices," Opt. Lett. 36, 772-774 (2011).

16. R. Iwanow, D. May-Arrioja, D. Christodoulides, G. Stegeman, Y. Min, and W. Sohler, "Discrete Talbot effect in waveguide arrays," Phys. Rev. Lett. 95, 053902 (2005).

17. F. Bloch, "Über die Quantenmechanik der Elektronen in Kristallgittern," Z. Phys. 52, 555-600 (1929).

18. R. Morandotti, U. Peschel, J. Aitchinson, H. Eisenberg, and Y. Silberberg, "Experimental observation of linear and nonlinear optical Bloch oscillations," Phys. Rev. Lett. 83, 4756-4759 (1999).

19. S. Longhi, M. Marangoni, M. Lobino, R. Ramponi, P. Laporta, E. Cianci, and V. Foglietti, "Observation of dynamic localization in periodically curved waveguide arrays," Phys. Rev. Lett. 96, 243901 (2006).

20. F. Dreisow, M. Heinrich, A. Szameit, S. Doering, S. Nolte, A. Tuennermann, S. Fahr, and F. Lederer, "Spectral resolved dynamic localization in curved fs laser written waveguide arrays," Opt. Express 16, 3474-3483 (2008).

21. P. Zhang, N. K. Efremidis, A. Miller, Y. Hu, and Z. Chen, "Observation of coherent destruction of tunneling and unusual beam dynamics due to negative coupling in three-dimensional photonic lattices," Opt. Lett. 35, 3252$3254(2010)$.

22. S. Longhi, "Imaging reconstruction in segmented waveguide arrays," Opt. Lett. 33, 473-475 (2008).

23. A. Szameit, F. Dreisow, M. Heinrich, T. Pertsch, S. Nolte, A. Tünnermann, E. Suran, F. Louradour, A. Barthelemy, and S. Longhi, "Image reconstruction in segmented femtosecond laser-written waveguide arrays," Appl. Phys. Lett. 93, 181109 (2008).

24. R. Keil, Y. Lahini, Y. Shechtman, M. Heinrich, R. Pugatch, F. Dreisow, S. Nolte, and A. Szameit, "Perfect imaging through a disordered waveguide lattice," Opt. Lett. 37, 809-811 (2012).

25. M. Heinrich, R. Keil, Y. Lahini, U. Naether, F. Dreisow, A. Tünnermann, S. Nolte, and A. Szameit, "Disorderenhanced nonlinear delocalization in segmented waveguide arrays," New J. Phys. 12, 073026 (2012).

26. K. Itoh, W. Watanabe, S. Nolte, and C. Schaffer, "Ultrafast processes for bulk modification of transparent materials," MRS Bull. 31, 620-625 (2006).

27. D. Christodoulides, F. Lederer, and Y. Silberberg, "Discretizing light behaviour in linear and nonlinear optical waveguide lattices," Nature 424, 817-823 (2003).

28. A. Jones, "Coupling of optical fibers and scattering in fibers," J. Opt. Soc. Am. 55, 261-271 (1965).

29. T. Schulte, S. Drenkelforth, G. Kleine Büning, W. Ertmer, J. Arlt, M. Lewenstein, and L. Santos, "Dynamics of Bloch oscillations in disordered lattice potentials," Phys. Rev. A 77, 023610 (2008).

30. I. Babushkin, A. Housakou, J. Herrmann, and Y. S. Kivshar, "Frequency-selective self-trapping and supercontinuum generation in arrays of coupled nonlinear waveguides," Opt. Express 15, 11978-11983 (2007).

31. F. Eilenberger, S. Minardi, A. Szameit, U. Röpke, J. Kobelke, K. Schuster, H. Bartelt, S. Nolte, L. Torner, F. Lederer, A. Tünnermann, and T. Pertsch, "Evolution dynamics of discrete-continuous light bullets," Phys. Rev. A 84, $013836(2011)$.

32. M. Kolesik and J. V. Moloney, "Nonlinear optical pulse propagation simulation: From maxwell's to unidirectional equations," Phys. Rev. E 70, 036604 (2004).

33. P. Kinsler, "Unidirectional optical pulse propagation equation for materials with both electric and magnetic responses," Phys. Rev. A 81, 023808 (2010).

34. M. Heinrich, A. Szameit, F. Dreisow, R. Keil, S. Minardi, T. Pertsch, S. Nolte, A. Tünnermann, and F. Lederer, "Erratum: Observation of three-dimensional discrete-continuous x waves in photonic lattices," Phys. Rev. Lett. 106, 029901 (2011).

35. G. Agrawal, Nonlinear Fiber Optics, 3rd ed. (Academic Press, 2001).

36. F. Eilenberger, M. de Sterke, and B. J. Eggleton, "Soliton mediated optical quantization in the transmission of one-dimensional photonic crystals," Opt. Express 18, 12708-12718 (2010).

37. T. Fukuda, S. Ishikawa, T. Fujii, K. Sakuma, and H. Hosoya, "Low-loss optical waveguides written by femtosecond laser pulses for three-dimensional photonic devices," Proc. SPIE 5339, 524-538 (2004).

38. H. Eisenberg, Y. Silberberg, R. Morandotti, and J. Aitchison, "Diffraction management," Phys. Rev. Lett. 85, 1863-1866 (2000).

39. Y. Vlasov, "Silicon integrated nanophotonics: Road from scientific explorations to practical applications," in Proceedings of CLEO/QELS, San Jose, CA, USA (2012).

\#174173 - \$15.00 USD Received 10 Aug 2012; revised 11 Sep 2012; accepted 12 Sep 2012; published 19 Nov 2012

(C) 2012 OSA

19 November 2012 / Vol. 20, No. 24 / OPTICS EXPRESS 27300 


\section{Introduction}

Integrated all-optical data-processing is a core research topic in telecommunication driven by the ever-increasing need for higher bandwidths $[1,2,3]$ and the inability of electronic components to handle terahertz data streams efficiently. Of particular interest for long-haul data transmission are so-called 3R stages, which are needed to retime, reshape, and regenerate pulse trains in an integrated, miniaturizable, and robust device and which are able to handle terahertz pulse trains. Signal reshaping is an intrinsically nonlinear process, in which low-power noise as well as peak-power variations need to be suppressed. The latter can be achieved by means of an optical limiting scheme [4,5], which imposes more loss for higher optical powers.

In the most general sense, an optical limiter is a system which features a decrease in transmission with growing input power. The perhaps most straightforward approach to this functionality would be to utilize absorption as natural counterpart to transmission. Nonlinear, i.e. intensity-dependent, absorption occurs in any transparent material if the photon density becomes sufficient to enable multiple photons to collectively overcome the band gap [4]. The general drawback of absorption is the deposition on energy in the device, which leads to heat accumulation and may even cause irreversible damage, especially in miniaturized and integrated systems. Thus it is desirable to instead divert light in an intensity-dependent manner. A well-known example for this kind of behavior is the interplay of diffraction and self-focusing in transparent media with positive Kerr coefficient, which can be used to mimic the action of a saturable absorber (e.g. for Kerr lens mode locking [6]). Similarly, optical limiting behavior may be achieved by utilizing nonlinearly self defocusing media [7].

Systems of evanescently coupled waveguides, so-called photonic lattices, have emerged as versatile platform for integrated optics [8]. In particular the interplay of discrete diffraction and nonlinearity has been subject of intensive research in recent years [9, 10]. Interestingly, photonic lattices support nonlinear localized states under both focusing [11] and defocusing [12] Kerr nonlinearity. These solitons resemble defect states residing on the transient index distribution induced by its intensity profile. In the context of telecommunications, they provide the possibility to route signals through photonic networks by purely optical means [13,14] and have reportedly even been utilized to transmit arbitrary intensity distributions [15].

Whereas under linear conditions, light injected into uniform photonic lattices undergoes ballistic spreading, various mechanisms may give rise to the revival of an initial input distribution. The discrete manifestation of the Talbot effect [16] reproduces transversely periodic input patterns in uniform lattices. In contrast, Bloch oscillations [17,18] and Dynamic localization [19,20] rely on specific transverse potential gradients to reconstruct arbitrary wave packets. Continuous longitudinal modulations of the lattice allow for the suppression of transverse broadening [21]. Finally, the underlying periodicity of photonic lattices also supports an exact reversal of diffraction by staggering the phase of a wave packet [22]. This so-called segmentation imaging perfectly reproduces any input intensity pattern [23] and even persists in lattices with arbitrary coupling disorder [24]. However, as all linear imaging mechanisms, segmentation imaging is susceptible to perturbations by nonlinear phase contributions [25].

In this paper we propose to utilize the nonlinear delocalization in segmented photonic lattices as an optical limiter. Due to the perfect imaging under linear conditions, such a device theoretically features unity unsaturated transmission. As an additional benefit, the diffractive propagation between input and output port leads to substantially decreased spectral distortions due to self phase modulation compared to the propagation through an isolated waveguide of equal length. The functionality of such a device is shown numerically and demonstrated experimentally in a photonic lattice realized in bulk fused silica with the femtosecond laser writing technique [26].

\#174173 - \$15.00 USD Received 10 Aug 2012; revised 11 Sep 2012; accepted 12 Sep 2012; published 19 Nov 2012

(C) 2012 OSA

19 November 2012 / Vol. 20, No. 24 / OPTICS EXPRESS 27301 


\section{Numerical investigations}

\subsection{Continuous wave $(\mathrm{CW})$ regime}

Assuming monochromatic conditions, the spatial evolution of a wave packet in photonic lattices is governed by a set of coupled differential equations [27] for the modal amplitudes $\varphi_{n}$ in the $n^{\text {th }}$ waveguide:

$$
-i \partial_{z} \varphi_{n}=C\left(\varphi_{n+1}+\varphi_{n-1}\right)+\left(\beta_{n}+\gamma\left|\varphi_{n}\right|^{2}\right) \varphi_{n}
$$

The mean propagation constant of the lattice has been removed by transformation into a copropagating reference frame. Deviations of the individual waveguides from this value are designated as $\beta_{n}$. The rate of mutual energy exchange between adjacent waveguides is determined by the coupling constant $C$, and the strength of the focusing Kerr nonlinearity is given by the coefficient $\gamma>0$. The total power

$$
P=\sum_{n}\left|\varphi_{n}(z)\right|^{2}
$$

is a conserved quantity of Eq. (1). To calculate the power-dependent transmission

$$
T=\left|\varphi_{0}(L)\right|^{2} / \sum_{n}\left|\varphi_{n}(L)\right|^{2},
$$

i.e. the power remaining in the excited waveguide $(n=0)$ after the total length $L$ of the lattice, we numerically solve the initial value problem of a $\delta$-like input field distribution $\varphi_{n}(0)=\delta_{n, 0}$. $\sqrt{P}$.

\subsubsection{Uniform lattice}

Let us first consider the case of a uniform lattice. According to the analytical solution of the linear system [28], the intensity in the excited channel decays with the Bessel function $J_{0}^{2}(2 C z)$ along the direction of propagation. The wave packet spreads symmetrically across the lattice in a V-shaped pattern [first column in Fig. 1(b)]. Although solitons exist for arbitrary powers in this one-dimensional setting, increasing the input power results in a sharp increase of $T$ around $P \approx 4 C / \gamma$ as the corresponding soliton becomes narrow enough to have a high overlap with the excitation [Fig. 1(a)]. This illustrates the saturable-absorber like characteristic of the system. However, the discrete soliton never becomes fully confined to a single site. The resulting diffractive background prevents $T$ from reaching 1 for finite powers.

\subsubsection{Negative defect}

The second column of Fig. 1 shows the corresponding behavior for a single, negatively detuned waveguide $\left(\beta_{0}=-4 C\right)$. Due to the presence of the defect state, a considerable fraction of light remains in the excited guide during linear propagation [Fig. 1(b)]. Since the negative defect state resides below the band, an increase in power decreases the degree of localization as phase matching occurs when the nonlinear propagation constant approaches the eigenvalues of the linear modes of the surrounding lattice [Fig. 1(a)]. The minimum transmission of $T_{\max }=28 \%$ occurs at $P=8.1 \mathrm{C} / \gamma$. Note that even for this strongly detuned lattice site, the linear defect state is sufficiently extended to limit the maximum transmission to only $T_{\max }=81 \%$ for the device length of $L=1.28 L_{C}$. Furthermore, the delocalization mechanism counteracts itself: As more and more light is radiated into the surrounding lattice, the detuning of the defect is compensated less efficiently. Finally, relocalization occurs as the nonlinear eigenvalue exceeds the upper edge of the band. Nevertheless, the negative defect site exhibits, albeit poorly, the fundamental behavior of an optical limiter.

\#174173 - \$15.00 USD Received 10 Aug 2012; revised 11 Sep 2012; accepted 12 Sep 2012; published 19 Nov 2012

(C) 2012 OSA

19 November 2012 / Vol. 20, No. 24 / OPTICS EXPRESS 27302 

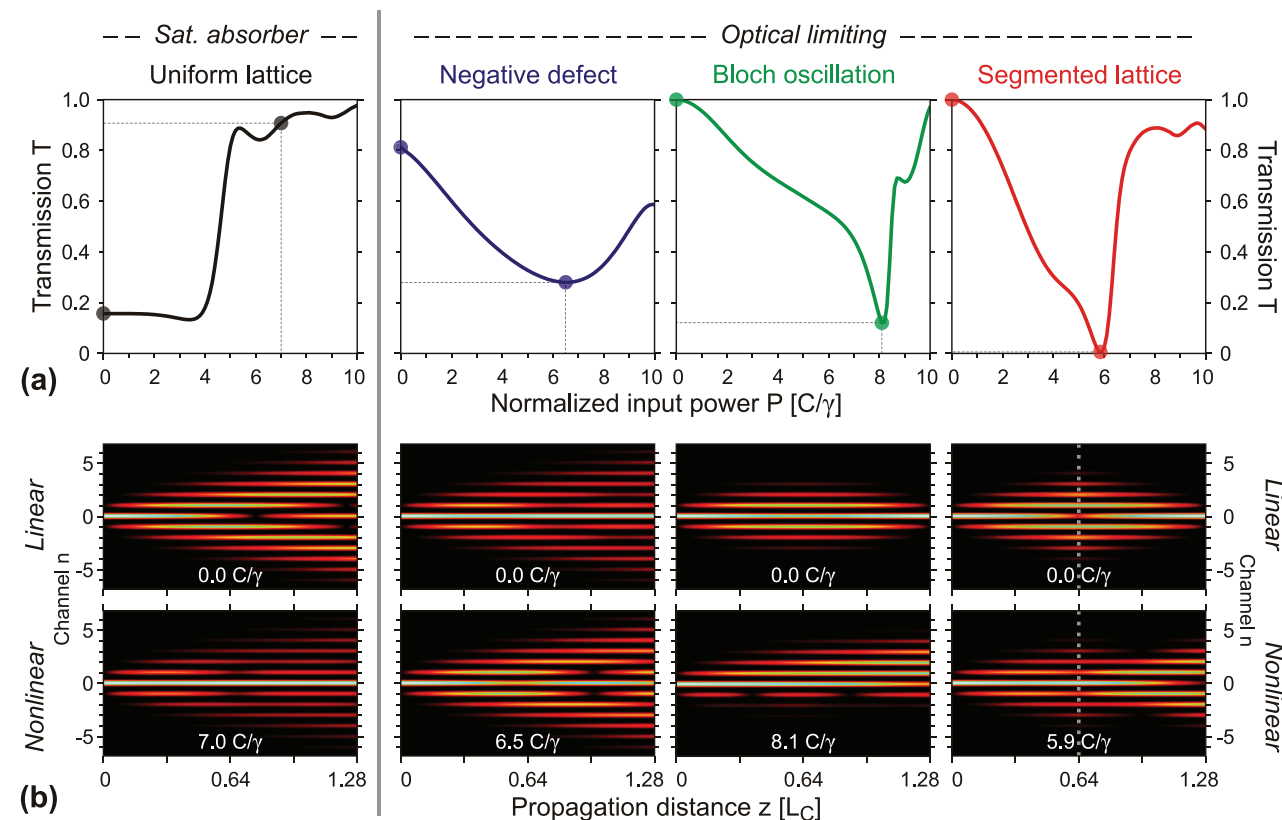

Fig. 1. (a) Power-dependence of the transmission of a uniform lattice [first column], a negative defect guide with $\beta_{0}=-4 C$ embedded in a uniform lattice [second column], a lattice with a transverse potential gradient that yields exactly one Bloch oscillation period [third column] and a segmented photonic lattice [fourth column]. In all cases, the sample length $L=1.28 L_{C}$ is identical. (b) Linear diffraction and exemplary nonlinear propagation patterns corresponding to the points marked in the respective $T(P)$ graphs in (a).

\subsubsection{Bloch oscillation}

Linear self-imaging allows for a theoretical maximum transmission of $T=1$ in the unsaturated regime. The third column of Fig. 1 shows this for a lattice with a transverse potential gradient of $\Delta \beta=\beta_{n+1}-\beta_{n}=2 \pi C / L$. This gradient causes Bloch oscillations [17] with a period equal to the device length, in our case $L=1.28 L_{C}$. Despite the transverse potential gradient the linear propagation through the lattice is symmetric [Fig.1(b)]. The nonlinearity breaks this symmetry, causes the wave packet to predominantly diffract towards higher $n$ and thus destroys the imaging property of the lattice. As a result, the transmission shows a steady decrease [Fig. 1(a)] to as low as $T_{\min }=12.1 \%$, eventually limited by the formation of a soliton at high powers. The nonlinear perturbation of Bloch oscillations therefore is a viable approach for an integrated optical limiter. However, from a practical point of view, their dependence on a specific lattice configuration is problematic as it renders the imaging susceptible to disorder [29]. The same holds true for self imaging in more intricate, $z$-dependent potentials such as Dynamic Localization, which generally are also more sensitive toward the wavelength [20] and thus less suitable for highbandwidth signals. 


\subsubsection{Segmentation imaging}

It was recently found that Segmentation Imaging, the reversal of discrete diffraction by means of imprinting a staggered phase, is impervious to coupling disorder [24]. The fourth column of Fig. 1 illustrates the performance of a segmented photonic lattice of $L=1.28 L_{C}$ for optical limiting. In the linear regime [Fig. 1(b)], light experiences ballistic diffractive spreading until $z=L / 2$. At this point the segmentation is accounted for by introducing an instantaneous phase flip:

$$
\varphi_{n}(L / 2) \rightarrow \mathrm{e}^{i n \pi} \varphi_{n}(L / 2)
$$

Subsequently the light converges back to the initially excited waveguide. This system features the maximum intermediate spreading of the wave packet compared to other linear imaging effects, as the outer lobes of ballistic propagation mark the edges of the region accessible by discrete diffraction for a given coupling constant. In the nonlinear regime, the additional phase contributions perturb the imaging effect. As the propagating wave packet becomes more localized in the uniform part of the lattice [Fig. 1(a)], a substantial fraction is converted into diffractive background at the segmentation [25]. Although the subsequent propagation is a rather complex and strongly power-dependent interplay of interference and nonlinearity, the overall transmission of the system at hand continuously decreases to less than one per cent of the initial value $\left(T_{\min }=0.6 \%\right)$. In conjunction with the perfect linear imaging, this constitutes a dynamic range $-\log _{10}\left(T_{\max }-T_{\min }\right)$ of approximately $22 \mathrm{~dB}$. Moreover, the power at which minimum transmission occurs is reduced to $P=5.9 \mathrm{C} / \gamma$ compared to the previously discussed Bloch lattice, which requires $P=8.1 C / \gamma$. Finally, such an optical limiting device [see schematic in Fig. 2] inherits the intrinsic robustness of segmentation imaging towards irregularly spaced waveguides [24].

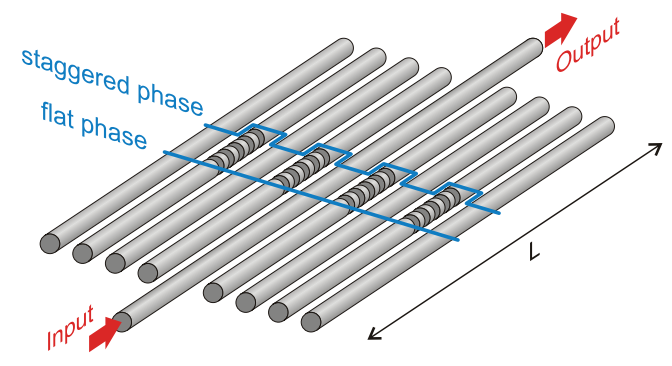

Fig. 2. Schematic of the device. At half the overall propagation length, every second waveguide is segmented such that light in these channels experiences a phase shift of $\pi$ compared to the neighboring channels. The resulting staggered phase front gives rise to perfect linear imaging between input and output port. For higher powers, the Kerr nonlinearity causes nonlinear delocalization at the segmented region, thus decreasing the fraction of light reaching the output port.

\#174173 - \$15.00 USD Received 10 Aug 2012; revised 11 Sep 2012; accepted 12 Sep 2012; published 19 Nov 2012

(C) 2012 OSA

19 November 2012 / Vol. 20, No. 24 / OPTICS EXPRESS 27304 


\subsection{Spatiotemporal simulations}

Rigorous simulations of the device functionality under short pulse excitation were carried out with a discrete-continuous unidirectional Maxwell-based method [30,31,32,33]. The set of coupled equations

$$
-i \operatorname{sgn}(\omega) \partial_{z} \Phi_{n}(z, \omega)=\left[\beta(\omega)-\omega \beta_{1}\right] \Phi_{n}(z, \omega)+C\left[\Phi_{n-1}(z, \omega)+\Phi_{n+1}(z, \omega)\right]+\frac{4}{3} \tilde{\gamma} \mathcal{P}_{n}(z, \omega)
$$

for the electric field amplitude $\Phi_{n}(z, t)=\phi_{n}(z, t) \cos \left(2 \pi c / \lambda_{0} t\right)$ in the $n^{\text {th }}$ waveguide of the array was solved in a reference frame moving with the inverse group velocity $\beta_{1}$. In addition to the nearest neighbor coupling $C$, this model also accounts for the dispersion $\beta(\omega)$ which is normal at the central wavelength $\lambda_{0}=800 \mathrm{~nm}$ of the pulse. The nonlinearity parameter $\tilde{\gamma}=0.00017 \mathrm{~mW}^{-1}$ was determined experimentally [34]. The nonlinear polarization in the $n^{\text {th }}$ waveguide $\mathcal{P}_{n}(z, \omega)$ arising from the Kerr effect, higher order nonlinear contributions and stimulated Raman scattering in the temporal domain is given by

$$
\mathcal{P}_{n}(z, t)=\Phi(z, t)\left[(1-f) \Phi_{n}^{2}(z, t)+f \int_{0}^{\infty} \Phi_{n}^{2}\left(z, t-t^{\prime}\right) \cdot h\left(t^{\prime}\right) \mathrm{d} t^{\prime}\right] .
$$

Here, the values for the Raman fraction $f$ and the time constants $\tau_{1,2}$ of the temporal response function

$$
h(t)=\frac{\tau_{1}^{2}+\tau_{2}^{2}}{\tau_{1} \tau_{2}^{2}} e^{-t / \tau_{2}} \sin \left(t / \tau_{1}\right) \quad \text { for } t \geq 0
$$

were taken from [35]. For reasons of clarity, technical issues such as segmentation loss and residual input chirp discussed in Section 3.2, are not included in the results presented here but have been evaluated in order to double check experimental results. The input pulse is of Gaussian shape with a full width at half maximum of $300 \mathrm{fs}$.

For pulsed excitation it is useful to introduce the overall transmission $\tilde{T}$ as the fraction of the injected pulse energy arriving at the output port:

$$
\tilde{T}=\int_{-\infty}^{+\infty}\left|\varphi_{0}(L, t)\right|^{2} \mathrm{~d} t / \sum_{n} \int_{-\infty}^{+\infty}\left|\varphi_{n}(L, t)\right|^{2} \mathrm{~d} t .
$$
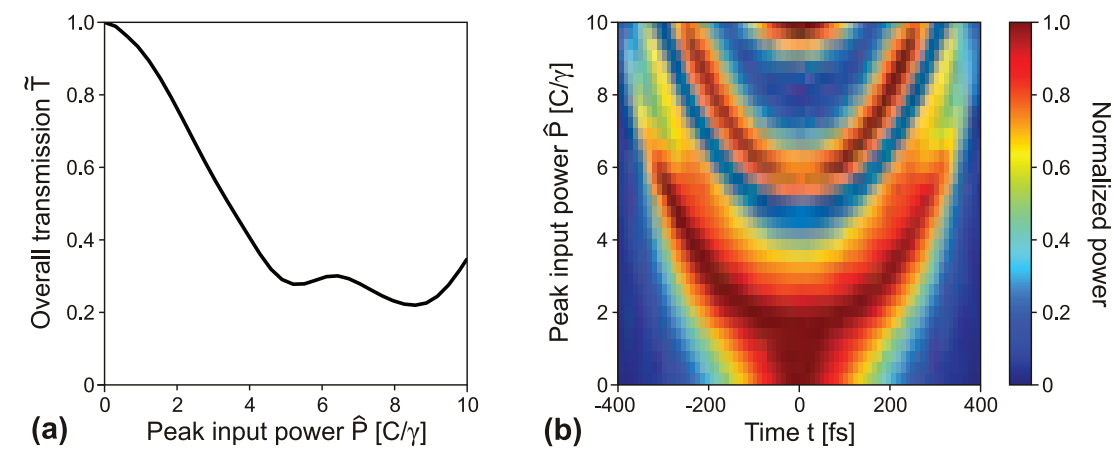

Fig. 3. (a) Calculated overall transmission $\tilde{T}$ of a Gaussian input pulse in dependence of the peak power $\hat{P}$. (b) Corresponding temporal pulse shape at the output port. The plot has been normalized with respect to the maximum value of each row. As $\hat{P}$ increases, the optical limiting causes the high-intensity part of the pulse to be diverted from the excited waveguide. Above $\hat{P} \approx 5 C \gamma$ relocalization after the segmented region leads to a more complex pulse shape. 

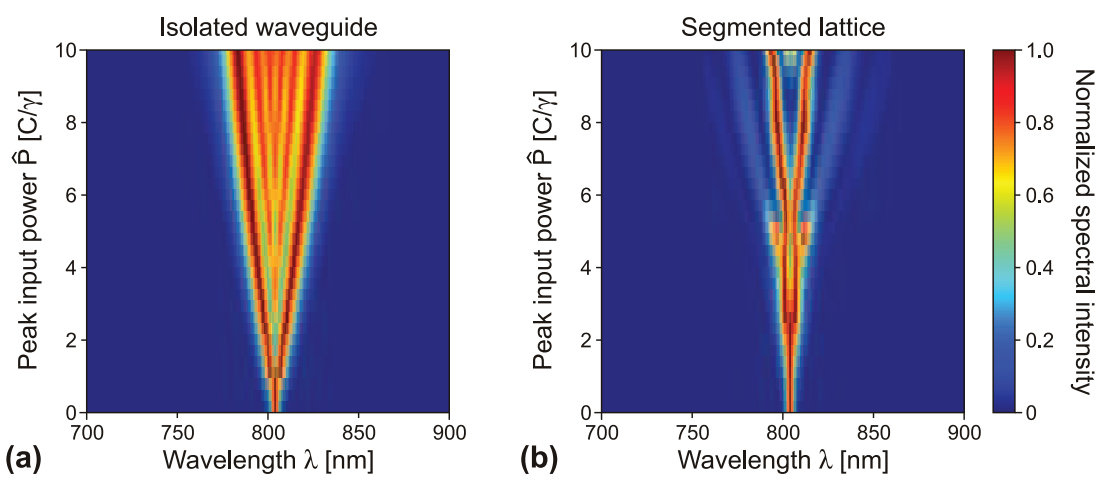

Fig. 4. Spectral stabilization: Simulated spectral broadening of $300 \mathrm{fs}$ Gaussian pulses in (a) an isolated waveguide and (b) in the device. For reasons of visibility, the plots have been normalized with respect to the maxima in each row.

Figure 3(a) summarizes the optical limiting behavior of the device. The reduction of the transmission with increasing input peak power $\hat{P}$ is clearly visible. Note that in first order approximation $\tilde{T}$ corresponds to the intensity-dependent $\mathrm{CW}$ transmission $T$ averaged over the pulse and hence does not maintain the strong attenuation shown in Fig. 2(a). Instead, a minimum overall transmission of $\tilde{T} \approx 25 \%$ is reached. This smoothed characteristic is fairly generic for nonlinear pulse dynamics unless solitary waves lead to optical quantization [36]. Being an artifact that arises from the definition of $\tilde{T}$ according to Eq. (8), it does not imply a flaw of the device's functionality.

Moreover our simulations show that the device's behavior under pulsed excitation corresponds well to the $\mathrm{CW}$ prediction for powers below the critical value of $P=4 C / \gamma$. This is illustrated by Fig. 3(b): Under linear conditions, the Gaussian shape of the pulse is maintained. As $\hat{P}$ is increased, the pulse shape is flattened as a growing fraction of power is diverted from the waveguide in the central part of the pulse. The nonlinear delocalization at $\hat{P} \approx 4.5 C / \gamma$ causes the high-intensity core of the pulse to be ejected almost completely, giving rise to a region of depleted intensity around its barycenter. Even higher input powers lead to pulse splitting as separate de-/ relocalization of parts of the pulse with different power levels produce more complex pulse shapes.

Analyzing the calculated output spectra, we found a drastic reduction of spectral distortions by virtue of the delocalized propagation of the pulse in the device. Figure 4(a) shows the powerdependent spectrum of pulses transmitted through an isolated waveguide of equal length. Self phase modulation almost immediately results in substantial broadening of the spectrum, and above $1 \mathrm{C} / \gamma$ the initially compact Gaussian envelope is replaced by a complex multi-peaked spectrum. In contrast, pulses passing through the device maintain a single-peaked spectrum up to $3 C / \gamma$ [Fig. 4(b)] and even beyond that the spectral width remains well below the isolated waveguide output.

In summary, our spatiotemporal simulations confirm optical limiting behavior for pulsed operation for peak powers $\hat{P} \lesssim 4 C / \gamma$. Spectral broadening is strongly suppressed in this regime. 


\section{Experimental results}

Using the femtosecond laser writing technique [26], we fabricated photonic lattices in a $10 \mathrm{~cm}$ long fused silica sample (Corning 7980). Optimum self imaging at the operational wavelength of $800 \mathrm{~nm}$ was achieved for a segmentation length of $5.2 \mathrm{~mm}$ with fill factor 0.5 . The waveguide separation of $34.5 \mu \mathrm{m}$ yielded a coupling constant of $C=0.2 \mathrm{~cm}^{-1}$, corresponding to an overall propagation length of $1.28 L_{C}$. For the characterization of our device we used a Ti:sapphire laser system (Spectra Physics Tsunami/Spitfire), delivering $290 \mathrm{fs}$ pulses with a repetition rate of $1 \mathrm{kHz}$ at a carrier wavelength of $\lambda=800 \mathrm{~nm}$. The beam was injected into a single waveguide of the lattice by means of a microscope objective $(N A=0.075)$.

\subsection{Optical limiting}

We recorded the output intensity distributions arising from launched average powers between $0<\bar{P} \leq 1.2 \mathrm{~mW}$ with a CCD camera (Basler A102-F). We determined the transmission of the device by simultaneously measuring the average power $\bar{P}$ in front and after the device with a dual-channel power meter (Newport 2835-C)

Figure 5(a) shows the observed power-dependent output pattern of the segmented lattice. For reasons of visibility each column has been normalized with respect to the total output power. In our experimental realization, loss in the segmented waveguides leads to imperfect segmentation imaging. Consequently, only $74 \%$ of the injected light are imaged back into the excited channel in the linear regime. Nevertheless, an increase in power clearly leads to a decrease in transmission $\tilde{T}$, whereas the amount of light contained in the surrounding waveguides increases notably. A plot of the independently measured power-dependent transmission of the system is shown in Fig. 5(b), clearly illustrating the limiting functionality in close resemblance with our numerical results up to a normalized peak power of $\hat{P}=\approx 3.5 \mathrm{C} / \gamma$.
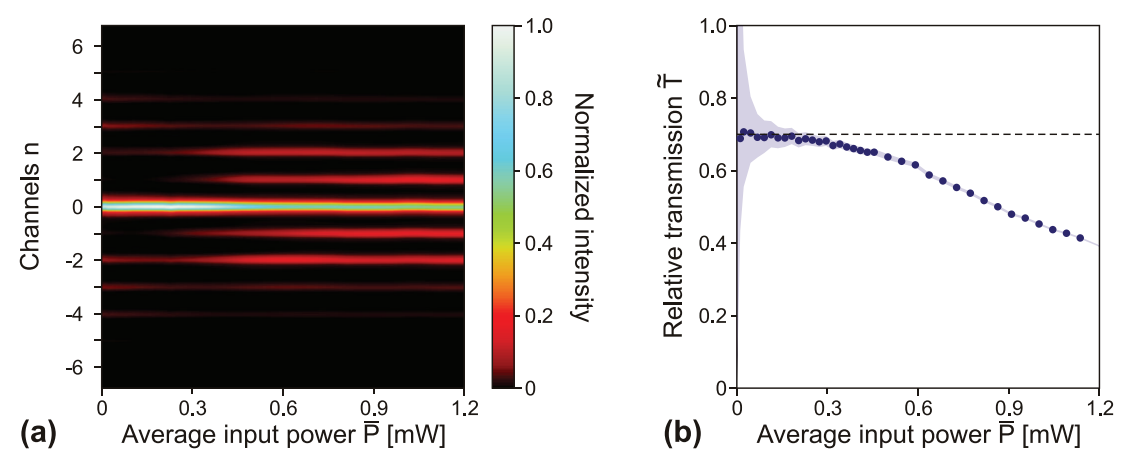

Fig. 5. Optical limiting: (a) Observed power-dependent output pattern of the segmented lattice for excitation with $280 \mathrm{fs}$ pulses. Each column has been normalized with respect to its total power. (b) Transmission through the device as measured by external power meters, corrected for propagation losses. The measurement error is indicated as shaded region around the data points. The non-unity transmission $(\tilde{T}=74 \%)$ for $\bar{P}=0$ can be attributed to the selective loss in the segmented waveguides. 

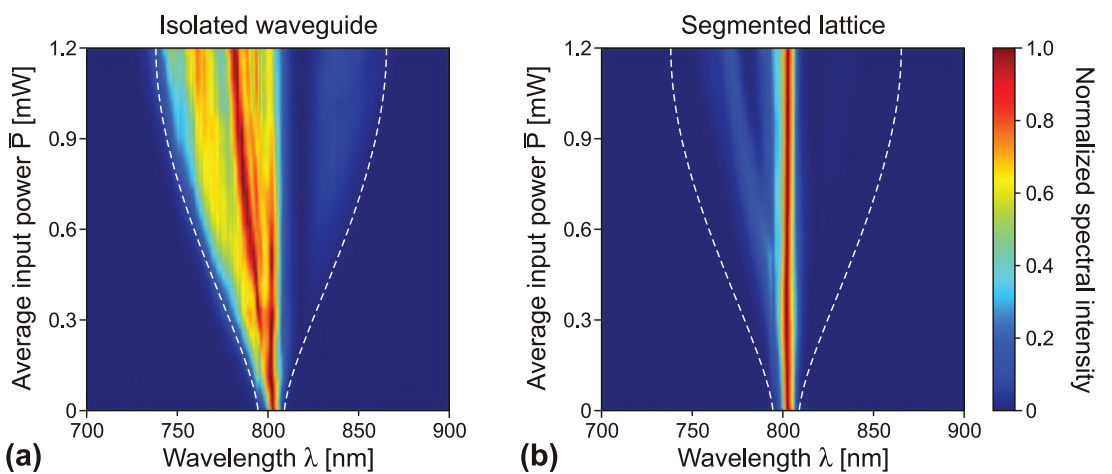

Fig. 6. Spectral stabilization: Measured spectral broadening of $280 \mathrm{fs}$ pulses propagating through (a) an isolated waveguide and (b) a segmented photonic lattice. For reasons of visibility, the plots have been normalized with respect to the maxima of each row. The dashed lines mark the outline of the spectral distribution in the isolated guide. We attribute the pronounced asymmetry of the spectra to the influence of higher-order chirp in the input pulse.

\subsection{Spectral stabilization}

To confirm the segmented lattice's mitigating influence on spectral distortions, we used an isolated waveguide with otherwise identical properties served as reference. We recorded the spectrum after propagation through the $100 \mathrm{~mm}$ long sample with a fiber-coupled spectrometer (OceanOptics USB-2000). Figure 6(a) clearly shows a substantial spectral broadening for input average powers $\bar{P}$ exceeding $0.1 \mathrm{~mW}$. The asymmetry of the nonlinear broadening predominantly arises from the input pulse's initial chirp, as confirmed by simulations.

In contrast, the spectrum of light transmitted through the segmented lattice remains almost unchanged throughout the investigated range of input powers [see Fig. 6(b)]. A weak blueshifted side lobe emerging above $\bar{P}=0.4 \mathrm{~mW}$ can be attributed to spectral changes occurring while the light is still contained in the excited guide during the initial phase of discrete diffraction.

\section{Discussion}

The femtosecond laser fabricated waveguide array used for our experiments demonstrates the expected principal behavior in good agreement with the theoretical predictions. Nevertheless, the performance of optical limiters based on segmentation imaging can be further optimized by currently available methods.

\subsection{Losses}

In the context of fully integrated devices, injection losses are of no relevance as the signal generally propagates inside waveguides. Similarly, the waveguide's propagation losses are omnipresent and hence should not be considered a specific property of the individual components of an optical circuit. Furthermore, even with fs laser inscription, losses as low as $0.05 \mathrm{~dB} / \mathrm{cm}$ have been reported [37]. Consequently, the impact of the realistic device exclusively arises in the segmented region.

In our experimental system, the segmentation introduces an attenuation of roughly $3 \mathrm{~dB}$ $(50 \%)$ in the segmented guides (odd $n$ ). As the wave packet extends over several segmented as well as unsegmented waveguides, the transmission of the device still reaches $\tilde{T}=74 \%$ in 
the linear regime. These losses can be reduced by replacing the segmentation with a continuous detuning. Alternatively, at the cost of an increased susceptibility to disorder, an appropriately designed kink in the waveguide array [38] can introduce a phase gradient of $\phi_{n}=n \pi$.

\subsection{Spectral stabilization}

In first order approximation, the ratio between spectral and spatial impact of nonlinearity can be chosen freely as the former depends exclusively on the intensity inside the individual waveguides, whereas the latter also scales with the coupling constant. Discrete diffraction leads to a broadening of the wave packet and hence reduces nonlinear contributions. Our device features a propagation length of $L / 2=0.64 L_{C}$ before the segmented region, which already results in a reduction of the peak intensity of $70 \%$. However, after a rapid decrease during the initial stages, the maximum intensity stabilizes due to the compact side lobes of the ballistic propagation pattern [see Fig. 7]. Consequently, increasing the device length over $\approx 1 L_{C}$ would only improve the suppression of nonlinear effects marginally.

In contrast, the fundamental character of segmentation imaging allows for an extension of this technique to two-dimensional square lattices [23], thereby exponentiating the number of waveguides the power is distributed over. With advanced fabrication techniques such as femtosecond laser inscription, the required volume structures can readily be fabricated.

\subsection{Miniaturization}

Recent advances in silicon integrated nanophotonics [39] have impressively demonstrated the miniaturization potential of integrated optical components down to the nanometer scale. The principle of segmentation imaging relies on the validity of the coupled mode approximation [22], which can be maintained for smaller dimensions by confining the waveguide's mode fields more tightly via an increased index contrast. This miniaturization also naturally lowers the required power as the effective area of the waveguide modes decreases.

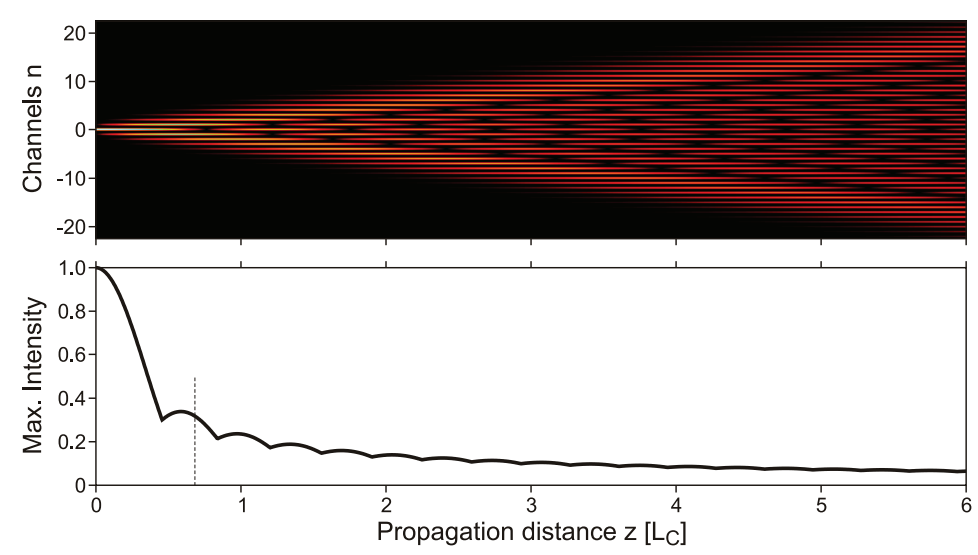

Fig. 7. Top: Ballistic propagation over $6 L_{C}$. Bottom: Reduction of the maximum intensity for discrete diffraction in the linear regime. The experimental propagation length of $L / 2=$ $0.64 L_{C}$ is indicated by a dashed line; it results in a $\approx 70 \%$ reduction of the peak intensity. 


\section{Conclusion}

We have introduced an integrated optical limiting device based on segmentation imaging in photonic lattices. Starting from unity transmission in the linear regime, nonlinear delocalization leads to a continuous decrease of the overall transmission for increasing input powers up to an extinction ratio of $22 \mathrm{~dB}$ under $\mathrm{CW}$ illumination.

Spatiotemporal simulations confirm the validity of the CW model for pulsed excitations. Since the functionality is based on the optical Kerr effect, the device's response follows the input intensity virtually instantaneously [9]. The diffractive propagation between input and output port distributes the light over multiple waveguides and thus substantially decreases spectral distortions due to self phase modulation. The functionality of the proposed device is demonstrated experimentally in femtosecond laser-written photonic lattices inscribed in fused silica.

Whereas the universal character of segmentation imaging allows for an extension of this technique to two-dimensional lattices to decrease the intermittent intensities even further, we have shown that such a device can readily be implemented in a one-dimensional setting and hence is accessible by conventional surface-bound fabrication techniques.

\section{Acknowledgments}

The authors gratefully acknowledge funding by the German Federal Ministry of Education and Research (ZIK 03Z1HN31 "ultra optics 2015") and the Thuringian Ministry for Education, Science and Culture (Research group "Spacetime", grant No. 11027-514). M.H. was supported by the German National Academy of Sciences Leopoldina (grant No. LPDS 2012-01), F.D. was financed by Graduate Research School OptiMi (B514-10061) and R.K. was supported by the Abbe School of Photonics.

\#174173 - \$15.00 USD Received 10 Aug 2012; revised 11 Sep 2012; accepted 12 Sep 2012; published 19 Nov 2012

(C) 2012 OSA

19 November 2012 / Vol. 20, No. 24 / OPTICS EXPRESS 27310 